Vol. 45 (1992) [61-69]

\title{
SEMIREGULAR GROUP DIVISIBLE DESIGNS WITH DUAL PROPERTIES
}

\author{
Alan Rahilly
}

\begin{abstract}
A construction method for group divisible designs is employed to construct (i) infinitely many non-symmetric semiregular group divisible designs whose duals are semiregular group divisible designs, and (ii) infinitely many transversal designs whose duals are group divisible 3-associate designs. A construction method for affine $\alpha$-resolvable balanced incomplete block designs is also given and illustrated.
\end{abstract}

\section{INTRODUCTION}

In this paper a construction method for group divisible designs (given in Section 3 ) is employed to construct semiregular group divisible designs having various sorts of uniform block intersections. (We refer to such group divisible designs as having "dual properties".) In particular, in Section 4 we construct infinitely many non-symmetric semiregular group divisible designs whose duals are semiregular group divisible designs and also infinitely many transversal designs whose duals are group divisible 3-associate designs [7]. Excluding constructions for self-dual transversal designs, constructions for infinite classes of semiregular group divisible designs whose duals are semiregular group divisible designs were largely unknown prior to the author's paper [8]. (The present paper, in fact, should be read as a sequel to [8]. Further details about what was known prior to [8] concerning the existence of semiregular group divisible designs with semiregular group divisible duals can be found in the introductory section of [8].) In Section 3 of this paper we also give a construction method for semiregular group divisible designs $\mathcal{G}^{*}$ whose duals are BIBDs (see Theorem 3(a)). The dual $\mathcal{G}^{* d}$ of $\mathcal{G}^{*}$ is then an affine $\alpha$-resolvable BIBD. This construction method is illustrated in Section 4 .

\section{Group Divisible Designs}

A tactical configuration ([3], p.4) with $v$ points, $b$ blocks, $r$ blocks on each point and $k$ points on each block is called a $(v, b, r, k)$-configuration. A $(v, b, r, k)$ configuration $(\mathcal{P}, \mathcal{B}, \mathcal{I})$ is said to be a group divisible design (GDD) if there is a partition

Received 17 January 1991

Copyright Clearance Centre, Inc. Serial-fee code: 0004-9729/92 \$A2.00+0.00. 
of $\mathcal{P}$ into "groups" $\mathcal{P}_{1}, \ldots, \mathcal{P}_{m_{2}}$, where $m_{2} \geqslant 2$, such that there are integers $m_{1} \geqslant 2$ and $\lambda_{1}$ and $\lambda_{2}$ such that

(a) $\left|\mathcal{P}_{i}\right|=m_{1}$ for all $i=1, \ldots, m_{2}$,

(b) any two points common to a group are on $\lambda_{1}$ blocks of $\mathcal{B}$,

(c) any two points in different groups are on $\lambda_{2}$ blocks of $\mathcal{B}$, and

(d) $\lambda_{1} \neq \lambda_{2}$.

We say that such a GDD $\mathcal{G}$ "has parameters $v, b, r, k ; m_{1}, m_{2} ; \lambda_{1}, \lambda_{2}$ " and that $\mathcal{P}_{1}, \ldots, \mathcal{P}_{m_{2}}$ is a "group division" of $\mathcal{G}$.

The parameters of a GDD satisfy the following equations

and

$$
\begin{gathered}
v r=b k \\
v=m_{1} m_{2} \\
\left(m_{1}-1\right) \lambda_{1}+m_{1}\left(m_{2}-1\right) \lambda_{2}=r(k-1) .
\end{gathered}
$$

It is well-known that group divisions can be exhaustively classified into the following mutually exclusive types:

1. Singular, for which $r=\lambda_{1}$.

2. Semiregular, for which $r>\lambda_{1}$ and

$$
r k=v \lambda_{2} .
$$

3. Regular, for which $r>\lambda_{1}$ and $r k>v \lambda_{2}$.

Since a GDD has a unique group division we can apply the terms "singular", "semiregular" and "regular" to GDDs as well as to group divisions.

For a semiregular GDD with parameters $v, b, r, k ; m_{1}, m_{2} ; \lambda_{1}, \lambda_{2}$ we have, from (2), (3) and (4), that

$$
r+\left(m_{1}-1\right) \lambda_{1}=m_{1} \lambda_{2} .
$$

REMARK. The basic results we have thus far mentioned concerning GDDs are given in [2].

A semiregular GDD with $\lambda_{1}=0$ is called a transversal design. For a transversal design we must have $k=m_{2}$.

It is possible for a GDD to possess two points which are incident with precisely the same blocks. Such points are said to be "repeated". It is easy to show that a GDD $\mathcal{G}$ has repeated points if and only if $\mathcal{G}$ is singular.

Next, let $k>0$ and $\bar{m}_{2} \geqslant 2$. An $\alpha$-resolution of a $(v, b, r, k)$-configuration $\mathcal{C}=$ $(\mathcal{P}, \mathcal{B}, \mathcal{I})$ is a partition $\mathcal{B}_{1}, \ldots, \mathcal{B}_{\bar{m}_{2}}$ of $\mathcal{B}$ such that each point of $\mathcal{P}$ is on precisely $\alpha$ 
blocks of each block class $\mathcal{B}_{i}$. For any $\alpha$-resolution $\mathcal{B}_{1}, \ldots, \mathcal{B}_{\overline{m_{2}}}$ it is straightforward to show that $\left|\mathcal{B}_{i}\right|=b / \bar{m}_{2}$ for each $i=1, \ldots, \bar{m}_{2}$ and that $\alpha=r / \bar{m}_{2}$. A 1-resolution is called a parallelism.

Let $\mathcal{G}=(\mathcal{P}, \mathcal{B}, \mathcal{I})$ be a semiregular GDD with parameters $v, b, r, k ; m_{1}, m_{2} ; \lambda_{1}, \lambda_{2}$. Bose and Connor [2] have shown that each block of $\mathcal{B}$ meets each group of $\mathcal{G}$ in $k / m_{2}$ points. Clearly, the groups of $\mathcal{G}$ form a $\left(k / m_{2}\right)$-resolution of $\mathcal{G}^{d}$ (the dual of $\mathcal{G}$ ). We also have

RESULT 1. Suppose $\mathcal{G}$ is a GDD with parameters $v, b, r, k ; m_{1}, m_{2} ; \lambda_{1}, \lambda_{2}$. If each block of $\mathcal{B}$ meets each group of $\mathcal{G}$ in $k / m_{2}$ points (that is, if the groups of $\mathcal{G}$ form a $\left(k / m_{2}\right)$-resolution of $\left.\mathcal{G}^{d}\right)$, then $\mathcal{G}$ is semiregular.

PROOF: There are $\Omega_{1}=\left(\begin{array}{c}m_{1} m_{2} \\ 2\end{array}\right)-\left(\begin{array}{c}m_{1} \\ 2\end{array}\right) m_{2}$ pairs of points $P, Q$ that can be chosen such that $P$ and $Q$ are in different groups of $\mathcal{G}$. Each block of $G$ accounts for $\Omega_{2}=\left(\begin{array}{c}k \\ 2\end{array}\right)-\left(\begin{array}{c}k / m_{2} \\ 2\end{array}\right) m_{2}$ such pairs of points. So we have $\Omega_{1} \lambda_{2}=\Omega_{2} b$ from which $\left(m_{1} m_{2}\right)^{2} \lambda_{2}=k^{2} b$ follows readily. But then (1) and (2) yield $v \lambda_{2}=r k$.

Remark. Result 1 is well known. A proof has been included since the author has not found one in the literature.

An $\alpha$-resolution $\mathcal{B}_{1}, \ldots, \mathcal{B}_{\bar{m}_{2}}$ of a $(v, b, r, k)$-configuration $\mathcal{G}$ is said to be an affine $\alpha$-resolution if $\mathcal{B}_{1}, \ldots, \mathcal{B}_{\bar{m}_{2}}$ is a (by Result 1 , necessarily semiregular) group division of $\mathcal{G}^{d}$. A BIBD with an affine parallellism with $t$ blocks in each affine parallel class and each pair of non-disjoint blocks meeting in $\mu$ points is called an $A R D(\mu, t)$.

We shall also need the following result.

Result 2. (Mitchell, [6]). Let $\mathcal{G}$ be a GDD such that $\mathcal{G}^{d}$ is also a GDD. Suppose that $\mathcal{G}$ has no repeated points or blocks (equivalently, that neither $\mathcal{G}$ nor $\mathcal{G}^{d}$ is a singular GDD). Then

(a) $\mathcal{G}$ and $\mathcal{G}^{d}$ are each semiregular, or

(b) $\mathcal{G}$ and $\mathcal{G}^{d}$ are each regular and $\mathcal{G}$ is symmetric.

Consider a semiregular GDD $\mathcal{G}$ with parameters $v, b, r, k ; m_{1}, m_{2} ; \lambda_{1}, \lambda_{2}$ whose dual is a semiregular GDD with parameters $b, v, k, r ; \bar{m}_{1}, \bar{m}_{2} ; \rho_{1}, \rho_{2}$. Equations (2), (4) and (5) for $\mathcal{G}^{d}$ yield $b=\bar{m}_{1} \bar{m}_{2}, r k=b \rho_{2}$ and

$$
k+\left(\bar{m}_{1}-1\right) \rho_{1}=\bar{m}_{1} \rho_{2} .
$$

There is one other equation which will be of use to us. Assuming $\mathcal{G}$ and $\mathcal{G}^{d}$ are semiregular GDDs with parameters as just given, let $A$ be an incidence matrix for $\mathcal{G}$. Now $A A^{t}$ has a unique eigenvalue distinct from 0 and $r k$ (namely $r-\lambda_{1}$ ) and $A^{t} A$ also has a unique such eigenvalue (namely $k-\rho_{1}$ ). Since the non-zero eigenvalues of $A A^{t}$ and $A^{t} A$ are the same, we have

$$
r-\lambda_{1}=k-\rho_{1} .
$$


Remarks. (a) Equation (7) has been derived by Mitchell [6]. For a more general context in which such an equation applies see [1].

(b) We also note that if $\mathcal{G}^{d}$ is a BIBD with index $\rho$, then an argument similar to that which led to (7) yields

$$
r-\lambda_{1}=k-\rho .
$$

A GDD is said to be self-dual if $\mathcal{G}^{d}$ is a GDD with the same parameters as $\mathcal{G}$. (Note that this is a weaker notion of self-duality than that which requires $\mathcal{G}$ and $\mathcal{G}^{d}$ to be isomorphic.)

\section{A Construction Method}

Suppose $\mathcal{G}=(\mathcal{P}, \mathcal{B}, \mathcal{I})$ is a semiregular GDD with parameters $v, b, r, k ; m_{1}, m_{2}$; $\lambda_{1}, \lambda_{2}$ and groups $\mathcal{P}_{i}, i=1, \ldots, m_{2}$. Suppose also that $\mathcal{G}^{\prime}=\left(\mathcal{P}^{\prime}, \mathcal{B}^{\prime}, \mathcal{I}^{\prime}\right)$ is a GDD with an $\alpha$-resolution $\mathcal{B}_{1}^{\prime}, \ldots, \mathcal{B}_{b}^{\prime}$ with $\left|\mathcal{B}_{i}^{\prime}\right|=\bar{m}_{1}^{\prime}$ for $i=1, \ldots, b$. Furthermore, suppose that the parameters of $\mathcal{G}^{\prime}$ are $v^{\prime}, \bar{m}_{1}^{\prime} b, \bar{m}_{1}^{\prime} r, k^{\prime} ; m_{1}, m_{2}^{\prime} ; \bar{m}_{1}^{\prime} \lambda_{1}, \bar{m}_{1}^{\prime} \lambda_{2}$ and that the groups of $\mathcal{G}^{\prime}$ are $\mathcal{P}_{i}^{\prime}, i=1, \ldots, m_{2}^{\prime}$. We also suppose $\mathcal{P} \cap \mathcal{P}^{\prime}=\emptyset$. We label the blocks of $\mathcal{G}$ by $B_{i}, i=1, \ldots, b$, and the blocks in $\mathcal{B}_{i}^{\prime}$ by $B_{i j}^{\prime}, j=1, \ldots, \bar{m}_{1}^{\prime}$, and define sets $B_{i j}^{*}$, $i=1, \ldots, b, j=1, \ldots, \bar{m}_{1}^{\prime}$ by

$$
B_{i j}^{*}=\left(B_{i j}^{\prime}\right) \cup\left(B_{i}\right)
$$

(Here $(B)$ stands for the set of points incident with block $B$.) Further, let $\mathcal{B}_{i}^{*}=\left\{B_{i j}^{*}\right.$ : $\left.j=1, \ldots, \bar{m}_{1}^{\prime}\right\}$.

Proposition 1. The incidence structure $\mathcal{G}^{*}=\left(\mathcal{P}^{*}, \mathcal{B}^{*}, \mathcal{I}^{*}\right)$, where $\mathcal{P}^{*}=$ $\mathcal{P} \cup \mathcal{P}^{\prime}, \mathcal{B}^{*}=\bigcup_{i=1}^{b} \mathcal{B}_{i}^{*}$ and $\mathcal{I}^{*}$ is given by set-theoretical inclusion, is a GDD with parameters $v+v^{\prime}, \bar{m}_{1}^{\prime} b, \bar{m}_{1}^{\prime} r, k+k^{\prime} ; m_{1}, m_{2}+m_{2}^{\prime} ; \bar{m}_{1}^{\prime} \lambda_{1}, \bar{m}_{1}^{\prime} \lambda_{2}$ and groups $\mathcal{P}_{1}, \ldots, \mathcal{P}_{m_{2}}$, $\mathcal{P}_{1}^{\prime}, \ldots, \mathcal{P}_{\mathbf{m}_{2}^{\prime}}^{\prime}$.

Proof: We establish the "balance" properties for $\mathcal{G}^{*}$ and leave the rest to the reader.

The substructure of $\mathcal{G}^{*}$ defined by $\mathcal{P}$ and $\mathcal{B}^{*}$ is an $\bar{m}_{1}^{\prime}$-multiple of a GDD isomorphic to $\mathcal{G}$ and with groups $\mathcal{P}_{1}, \ldots, \mathcal{P}_{m_{2}}$, and the substructure of $\mathcal{G}^{*}$ defined by $\mathcal{P}^{\prime}$ and $\mathcal{B}^{*}$ is isomorphic to $\mathcal{G}^{\prime}$ and has groups $\mathcal{P}_{1}^{\prime}, \ldots, \mathcal{P}_{m_{2}^{\prime}}^{\prime}$. So two points in the same $\mathcal{P}_{i}$, or in the same $\mathcal{P}_{i}^{\prime}$, are on $\bar{m}_{1}^{\prime} \lambda_{1}$ blocks of $\mathcal{G}^{*}$. Also, two points, one from $\mathcal{P}_{i}$ and one from $\mathcal{P}_{j}$, where $i \neq j$, are on $\bar{m}_{1}^{\prime} \lambda_{2}$ blocks of $\mathcal{G}^{*}$ and similarly for a pair of points, one from $\mathcal{P}_{i}^{\prime}$ and one from $\mathcal{P}_{j}^{\prime}$.

Consider $P \in \mathcal{P}$ and $P^{\prime} \in \mathcal{P}^{\prime} . P$ is on $r$ blocks of $\mathcal{G}$, say $B_{i_{1}}, \ldots, B_{i_{r}}$. So the set of blocks of $\mathcal{G}^{*}$ which are incident with $P$ is $\bigcup_{l=1}^{r} \mathcal{B}_{i_{l}}^{*}$. But $P^{\prime}$ is on $\alpha=\bar{m}_{1}^{\prime} r / b=\bar{m}_{1}^{\prime} k / v$ 
blocks of each $\mathcal{B}_{i}^{\prime}$ and hence is on this number of blocks of each $\mathcal{B}_{i}^{*}$. Therefore $P$ and $P^{\prime}$ are common to $\bar{m}_{1}^{\prime} r k / v=\bar{m}_{1}^{\prime} \lambda_{2}$ blocks of $\mathcal{G}^{*}$.

REMARKS. (a) Since $\mathcal{G}$ is not singular, neither $\mathcal{G}^{\prime}$ nor $\mathcal{G}^{*}$ is singular.

(b) If $\mathcal{G}$ is a $(v, b, r, k, \lambda)$-design and $\mathcal{G}^{\prime}$ is a $\left(v, \bar{m}_{1}^{\prime} b, \bar{m}_{1}^{\prime} r, k, \bar{m}_{1}^{\prime} \lambda\right)$-design with an $\alpha$-resolution $\mathcal{B}_{1}^{\prime}, \ldots, \mathcal{B}_{b}^{\prime}$ with $\left|\mathcal{B}_{i}^{\prime}\right|=\bar{m}_{1}^{\prime}$ for $i=1, \ldots, b$, then the construction method above yields a (semiregular) GDD with two groups of points (namely $\mathcal{P}$ and $\mathcal{P}^{\prime}$ ). In this situation the parameters of $\mathcal{G}^{*}$ are $2 v, \bar{m}_{1}^{\prime} b, \bar{m}_{1}^{\prime} r, 2 k ; v, 2 ; \bar{m}_{1}^{\prime} \lambda,\left(\bar{m}_{1}^{\prime} r k\right) / v$.

PROPOSITION 2. $\mathcal{G}^{*}$ is semiregular if and only if $\mathcal{G}^{\prime}$ is semiregular.

Proof:

$$
\begin{aligned}
G^{*} \text { is semiregular } & \Longleftrightarrow\left(v+v^{\prime}\right) \bar{m}_{1}^{\prime} \lambda_{2}=\bar{m}_{1}^{\prime} r\left(k+k^{\prime}\right) \\
& \Longleftrightarrow v^{\prime}\left(\bar{m}_{1}^{\prime} \lambda_{2}\right)=\left(\bar{m}_{1}^{\prime} r\right) k^{\prime} \quad \text { (since } \mathcal{G} \text { is semiregular) } \\
& \Longleftrightarrow \mathcal{G}^{\prime} \text { is semiregular. }
\end{aligned}
$$

The most obvious way to apply our construction method to obtain semiregular GDDs is to let $v=v^{\prime}$ (whence $m_{2}=m_{2}^{\prime}$ ), $k=k^{\prime}, \mathcal{G}$ be a semiregular GDD with an $\alpha$-resolution with $\bar{m}_{1}^{\prime}$ blocks in each $\alpha$-resolution class and $\mathcal{G}^{\prime}$ be an $\bar{m}_{1}^{\prime}$-multiple of a GDD isomorphic to $\mathcal{G}$. In this situation the $\alpha$-resolution of $\mathcal{G}$ induces an $\alpha$-resolution of $\mathcal{G}^{\prime}$ with $\overline{\boldsymbol{m}}_{1}^{\prime}$ blocks in each $\alpha$-resolution class.

REMARKs. (a) Our construction method generalises the dual form of the construction method considered in Theorem 7(ii) of Mavron [5].

(b) Semiregular GDDs with an $\alpha$-resolution can be constructed from a BIBD and a GDD with a parallelism by using the method of [9]. The method of [8, Section 4], can also be used to construct semiregular GDDs with an $\alpha$-resolution.

(c) Suppose we apply our construction method with $\mathcal{G}$ a regular GDD and $m_{2}=$ $m_{2}^{\prime}$. Then, for the constructed $\mathcal{G}^{*}$, we have that

(i) two points common to a group of $\mathcal{G}$ or to a group of $\mathcal{G}^{\prime}$ are on $\bar{m}_{1}^{\prime} \lambda_{1}\left(\neq \bar{m}_{1}^{\prime} \lambda_{2}\right)$ blocks of $\mathcal{G}^{*}$,

(ii) two points in different groups of $\mathcal{G}$ or of $\mathcal{G}^{\prime}$ are on $\bar{m}_{1}^{\prime} \lambda_{2}$ blocks of $\mathcal{G}^{*}$, and

(iii) $|\mathcal{P}|=\left|\mathcal{P}^{\prime}\right|$ and a point of $\mathcal{P}$ and a point of $\mathcal{P}^{\prime}$ are on $\left(\bar{m}_{1}^{\prime} r k\right) / v\left(\neq \bar{m}_{1}^{\prime} \lambda_{2}\right)$ blocks of $\mathcal{G}^{*}$.

Thus, in the terminology of [7], $\mathcal{G}^{*}$ is a "group divisible 3-associate design".

Next, we consider the situation in which the $\alpha$-resolution of $\mathcal{G}^{\prime}$ is an affine $\alpha$ resolution. When this is so $\mathcal{G}^{\prime d}$ is a semiregular GDD. Since $\mathcal{G}^{\prime}$ is not singular we 
have, by Result 2 , that $\mathcal{G}^{\prime}$ is also semiregular, and so by Proposition $2, \mathcal{G}^{*}$ is semiregular. Since the construction of semiregular GDDs with dual properties is our aim, the following theorem is our main result.

THEOREM 3. Suppose the $\alpha$-resolution of $\mathcal{G}^{\prime}$ is an affine $\alpha$-resolution of $\mathcal{G}^{\prime}$ with each pair of blocks in the same $\alpha$-resolution class meeting in $p_{1}^{\prime}$ points and each pair of blocks in different $\alpha$-resolution classes meeting in $\rho_{2}^{\prime}$ points.

(a) If $\mathcal{G}^{d}$ is an affine $\left(k / m_{2}\right)$-resolvable $(b, v, k, r, \rho)$-design, then $\mathcal{G}^{* d}$ is an affine $\left(\left(k+k^{\prime}\right) /\left(m_{2}+m_{2}^{\prime}\right)\right)$-resolvable $\left(\bar{m}_{1}^{\prime} b, v+v^{\prime}, k+k^{\prime}, \bar{m}_{1}^{\prime} r, \rho+\rho_{2}^{\prime}\right)$ design.

(b) If $\mathcal{G}^{d}$ is a semiregular GDD with parameters $b, v, k, r ; \bar{m}_{1}, \bar{m}_{2} ; \rho_{1}, \rho_{2}$ and groups $\mathcal{B}_{\gamma}=\left\{B_{(\gamma-1) \bar{m}_{1}+\beta}: \beta=1, \ldots, \bar{m}_{1}\right\}, \gamma=1, \ldots, \bar{m}_{2}$, then $\mathcal{G}^{* d}$ is a semiregular GDD with parameters $\bar{m}_{1}^{\prime} b, v+v^{\prime}, k+k^{\prime}, \bar{m}_{1}^{\prime} r ; \bar{m}_{1}^{\prime} \bar{m}_{1}, \bar{m}_{2} ; \rho_{1}+$ $\rho_{2}^{\prime}, \rho_{2}+\rho_{2}^{\prime}$ and groups $\bigcup_{f=(i-1) \bar{m}_{1}+1}^{i \bar{m}_{1}} \mathcal{B}_{f}^{*}, i=1, \ldots, \bar{m}_{2}$.

Proof: We shall prove only Part (b). Part (a) can be established in a similar manner. (At the point in the proof of Part (b) where (7) is applied to $\mathcal{G}$ one applies (8).)

(b) For convenience we denote $\mathcal{B}_{j}^{*}$ by $\mathcal{B}^{*}(i, j)$, where $i$ and $j$ are the unique integers such that $f=(i-1) \bar{m}_{1}+j, 1 \leqslant i \leqslant \bar{m}_{2}$ and $1 \leqslant j \leqslant \bar{m}_{1}$.

Let $B^{*} \in \mathcal{B}^{*}(i, j)$ and $B^{* *} \in \mathcal{B}^{*}(h, l)$. We consider three cases.

CASE 1: $i=h, j=l$

$B^{*}$ and $B^{* *}$ meet in $k$ points of $\mathcal{P}$ and $\rho_{1}^{\prime}$ points of $\mathcal{P}^{\prime}$.

CASE 2: $i=h, j \neq l$

$B^{*}$ and $B^{* *}$ meet in $\rho_{1}$ points of $\mathcal{P}$ and $\rho_{2}^{\prime}$ points of $\mathcal{P}^{\prime}$.

CASE 3: $i \neq h$

$B^{*}$ and $B^{* *}$ meet in $\rho_{2}$ points of $\mathcal{P}$ and $\rho_{2}^{\prime}$ points of $\mathcal{P}^{\prime}$.

Now

$$
\begin{array}{rlrl}
\rho_{2}^{\prime}-\rho_{1}^{\prime} & =\frac{k^{\prime}-\rho_{1}^{\prime}}{\bar{m}_{1}^{\prime}} & & \left((6) \text { applied to } \mathcal{G}^{\prime}\right) \\
& =\frac{\bar{m}_{1}^{\prime} r-\bar{m}_{1}^{\prime} \lambda_{1}}{\bar{m}_{1}^{\prime}} & & \left((7) \text { applied to } \mathcal{G}^{\prime}\right) \\
& =r-\lambda_{1} & \\
& =k-\rho_{1} & & ((7) \text { applied to } \mathcal{G}) .
\end{array}
$$

Clearly we have $k+\rho_{1}^{\prime}=\rho_{1}+\rho_{2}^{\prime}$ and so $\mathcal{G}^{* d}$ is a GDD with groups $\bigcup_{j=1}^{\bar{m}_{1}} \mathcal{B}^{*}(i, j)=$ 
$\bigcup_{f=(i-1) \bar{m}_{1}+1}^{i \bar{m}_{1}} \mathcal{B}_{f}^{*}$. But then $\bar{m}_{1}^{\prime} b\left(\rho_{2}+\rho_{2}^{\prime}\right)=\bar{m}_{1}^{\prime} r\left(k+k^{\prime}\right)$ is easily established, since $\mathcal{G}^{d}$
and $\mathcal{G}^{\prime d}$ are semiregular. Hence, $\mathcal{G}^{* d}$ is semiregular. Remarks. (a) The construction of Theorem 3(a) generalises the dual form of that of Theorem 7(iii) of Mavron [5]. Note that we have used a different method of proof to Mavron in generalising his results.

(b) The only construction methods for affine $\alpha$-resolvable BIBDs with $\alpha>1$ known to the author are

(i) that of Shrikhande and Raghavarao [9],

(ii) a construction method in [8, see p. 170], and

(iii) that of Theorem 3(a) above.

\section{ILLUSTRATIONS}

In this section we give some constructions of semiregular GDDs with dual properties that illustrate the method developed in Section 3. Throughout this section a self-dual GDD with parameters

$$
q^{n+2}, q^{n+2}, q^{n+1} \sigma, q^{n+1} \sigma ; q, q^{n+1} ; q^{n+1} \frac{\sigma(\sigma-1)}{q-1}, q^{n} \sigma^{2}
$$

will be referred to as an $\mathcal{S}(q, n, \sigma) . \mathcal{S}(q, n, 1)$ 's are transversal designs and are known to exist for $q$ any prime power and all $n \geqslant 0$. Given an $\mathcal{S}(q, n, 1)$ and a $(q, \sigma,(\sigma(\sigma-1)) /(q-1))$-design we can apply the method of [8, Section 4], to construct an $\mathcal{S}(q, n, \sigma)$.

Consider an affine $\sigma$-resolvable $\left(q^{n+1},\left(q\left(q^{n+1}-1\right)\right) /(q-1),\left(\sigma\left(q^{n+1}-1\right)\right) /\right.$ $\left.(q-1), q^{n} \sigma,\left(\sigma\left(q^{n} \sigma-1\right)\right) /(q-1)\right)$-design $\mathcal{G}^{d}$. The dual $\mathcal{G}$ of $\mathcal{G}^{d}$ is a semiregular GDD with parameters

$$
\frac{q\left(q^{n+1}-1\right)}{q-1}, q^{n+1}, q^{n} \sigma, \frac{\sigma\left(q^{n+1}-1\right)}{q-1} ; q, \frac{q^{n+1}-1}{q-1} ; q^{n} \frac{\sigma(\sigma-1)}{q-1}, q^{n-1} \sigma^{2} .
$$

(Such affine $\sigma$-resolvable designs can be constructed using the method of [9].) With $\mathcal{G}^{\prime}$ an $\mathcal{S}(q, n, \sigma)$, Theorem $3\left(\right.$ a) yields that $\mathcal{G}^{* d}$ is an affine $\sigma$-resolvable design with parameters

$$
\left(q^{n+2}, \frac{q\left(q^{n+2}-1\right)}{q-1}, \frac{\sigma\left(q^{n+2}-1\right)}{q-1}, q^{n+1} \sigma, \frac{\sigma\left(q^{n+1} \sigma-1\right)}{q-1}\right) .
$$

Next, given an $\mathcal{S}(q, n, 1)$ and a self-dual semiregular GDD $\overline{\mathcal{G}}$ with parameters $v, v, k, k ; q, m_{2} ; \lambda_{1}, \lambda_{2}$, it is possible to construct a self-dual semiregular GDD $\mathcal{G}$ with parameters $q^{n+1} v, q^{n+1} v, q^{n+1} k, q^{n+1} k ; q, q^{n+1} m_{2} ; q^{n+1} \lambda_{1}, q^{n+1} \lambda_{2}$ (see [8, Section 4]). 
Using an $\mathcal{S}(q, n+1,1)$ with $\overline{\mathcal{G}}$ in this way yields a self-dual semiregular GDD $\mathcal{G}^{\prime}$ with parameters $q^{n+2} v, q^{n+2} v,, q^{n+2} k, q^{n+2} k ; q, q^{n+2} m_{2} ; q^{n+2} \lambda_{1}, q^{n+2} \lambda_{2}$. Propositions 1 and 2 and Theorem 3(b) allow us to infer that $\mathcal{G}^{*}$ is a semiregular GDD whose dual $\mathcal{G}^{* d}$ is also a semiregular GDD. In this situation $\mathcal{G}^{*}$ is not symmetric. If $\overline{\mathcal{G}}$ is a transversal design, then $\mathcal{G}^{*}$ is a transversal design whose dual is a semiregular GDD, but not a transversal design. Examples for $\overline{\mathcal{G}}$ which are not transversal designs are known with $v=\bar{q}^{h}\left(\left(\bar{q}^{h}-1\right) /(\bar{q}-1)\right), k=\bar{q}^{h-1} \bar{\sigma}\left(\left(\bar{q}^{h}-1\right) /(\bar{q}-1)\right), m_{2}=\left(\bar{q}^{h}-1\right) /(\bar{q}-1), \lambda_{1}=$ $\bar{q}^{h-1} \bar{\sigma}\left(\left(\bar{q}^{h-1} \bar{\sigma}-1\right) /(\bar{q}-1)\right)$ and $\lambda_{2}=\bar{q}^{h-2} \bar{\sigma}^{2}\left(\left(\bar{q}^{h}-1\right) /(\bar{q}-1)\right)$, where $\bar{q}$ is any prime power, $h \geqslant 2$ and there is a $(\bar{q}, \bar{\sigma},(\bar{\sigma}(\bar{\sigma}-1)) / \bar{q}-1)$-design (possibly a $(\bar{q}, 1,0)$ "design"); see [8, pp. 170-1]. (Examples with these parameters with $\bar{\sigma}=1$ are also given in [4].)

Finally, consider a semiregular GDD $\mathcal{G}$ such that $\mathcal{G}^{d}$ is a singular GDD and a semiregular GDD $\mathcal{G}^{\prime}$ whose dual is a semiregular GDD. Suppose that the parameters of $\mathcal{G}$ and $\mathcal{G}^{\prime}$ are such that the construction method of Section 3 can be applied to produce a semiregular GDD $\mathcal{G}^{*}$. An argument of a similar nature to that which established Theorem $3(\mathrm{~b})$ shows that $\mathcal{G}^{* d}$ is a group divisible 3 -associate design.

Let $\mathcal{D}$ be an $A R D\left(q^{H-2}, q\right)$, where $q$ is a prime power and $H \geqslant 2$. Also, let $\mathcal{G}$ be a $q^{n+1-H_{-}}$-multiple of $\mathcal{D}^{d}$, where $n \geqslant H$. Then $\mathcal{G}$ is a semiregular GDD with parameters

$$
\frac{q\left(q^{H}-1\right)}{q-1}, q^{n+1}, q^{n}, \frac{q^{H}-1}{q-1} ; q, \frac{q^{H}-1}{q-1} ; 0, q^{n-1}
$$

and $\mathcal{G}^{d}$ is a singular GDD with parameters

$$
q^{n+1}, \frac{q\left(q^{H}-1\right)}{q-1}, \frac{q^{H}-1}{q-1}, q^{n} ; q^{n+1-H}, q^{H} ; \frac{q^{H}-1}{q-1}, \frac{q^{H-1}-1}{q-1}
$$

(These results concerning the parameters of $\mathcal{G}$ and $\mathcal{G}^{d}$ follow from the expressions for the parameters of an $A R D(\mu, m)$ as given on p.73 of [3].) Let $\mathcal{G}^{\prime}$ be a semiregular GDD with parameters

$$
q^{n+2}\left(\frac{q^{h}-1}{q-1}\right), q^{h+n+1}, q^{h+n}, q^{n+1}\left(\frac{q^{h}-1}{q-1}\right) ; q, q^{n+1}\left(\frac{q^{h}-1}{q-1}\right) ; 0, q^{h+n-1}
$$

whose dual is a semiregular $G D D$ with parameters

$$
\begin{array}{r}
q^{h+n+1}, q^{n+2}\left(\frac{q^{h}-1}{q-1}\right), q^{n+1}\left(\frac{q^{h}-1}{q-1}\right), q^{h+n} ; q^{h}, q^{n+1} ; q^{n+1}\left(\frac{q^{h-1}-1}{q-1}\right) \\
q^{n}\left(\frac{q^{h}-1}{q-1}\right) .
\end{array}
$$

Such a $\mathcal{G}^{\prime}$ exists for each prime power $q, h \geqslant 2$ and $n \geqslant 0$ (see [8, p.172]). The parameters of $\mathcal{G}$ and $\mathcal{G}^{\prime}$ are such that we can use the construction of this paper to 
produce a transversal design $\mathcal{G}^{*}$ whose dual is a group divisible 3 -associate design. The intersection numbers for blocks of $\mathcal{G}^{*}$ are

$$
\frac{q^{H}-1}{q-1}+q^{n+1}\left(\frac{q^{h-1}-1}{q-1}\right), \frac{q^{H}-1}{q-1}+q^{n}\left(\frac{q^{h}-1}{q-1}\right) \text { and } \frac{q^{H-1}-1}{q-1}+q^{n}\left(\frac{q^{h}-1}{q-1}\right) \text {. }
$$

\section{References}

[1] H. Beker, 'Strongly divisible 1-designs', Geom. Dedicata 8 (1979), 299-316.

[2] R.C. Bose and W.S. Connor, 'Combinatorial properties of group divisible incomplete block designs', Ann. Math. Statist. 23 (1952), 367-383.

[3] P. Dembowski, Finite geometries (Springer-Verlag, Berlin, Heidelberg, New York, 1968).

[4] D. Jungnickel, 'On automorphism groups of divisible designs', Canad. J. Math. 34 (1982), 257-297.

[5] V.C. Mavron, 'Constructions for resolvable and related designs', Aequationes Math. 23 (1981), 131-145.

[6] C. Mitchell, 'Group divisible designs with dual properties', Mitt. Math. Sem. Giessen 165 (1984), 105-117.

[7] D. Raghavarao, 'A generalization of group divisible designs', Ann. Math. Statist. 31 (1960), 756-771.

[8] Alan Rahilly, 'Semiregular group divisible designs whose duals are semiregular group divisible', Australas. J. Combin. 2 (1990), 157-179.

[9] S.S. Shrikhande and D. Raghavarao, 'A method of construction of incomplete block designs', Sankhyā Ser. A 25 (1963), 399-402.

Department of Mathematics

The University of Queensland

Queensland 4072

Australia 